Cimmerio per mezzo d' una croce obliqua assai ben delineata (Padargus ed Harpasus di Lowell). Il Lacus Tithonius fu nettamente risoluto in un triangoletto col vertice ottuso al nord, così come lo disegnò Lowell nel i 894, e finalmente anche sulla Gransirte si osservarono due linee di maggior ombra, una longitudinale dalla punta del golfo alla base d' Ellade, l' altra trasversale, dalla punta della Piccola Sirte alla Terra di Yao.

Teramo 1897 Febbraio r.

V. Cerulli.

\title{
Bemerkungen zu Herrn Prof. Schur's Abhandlung „Neue Untersuchungen über den Verlauf der systematischen Fehler bei Distanzmessungen " in A. N. 3399.
}

Ich will im Folgenden auf Herrn Prof. Schur's Arbeit „Neue Untersuchungen...\& (A. N. 3399) kurz eingehen und zeigen, dass dieselben zu einer schönen Bestätigung der von mir. (A. N. 3397-98) abgeleiteten Resultate führt.

Prof. Schur unternimmt es, die systematischen Fehler seiner Messungen des Löwenbogens abzuleiten, ohne eine bestirnmte Annahme über ihre Form zu Grunde zu legen. Er fasst dazu die gemessenen Distanzen ihrer Grösse nach in 8 Gruppen zusammen und führt für jede Gruppe je einen systematischen Fehlerwerth (I), (2), .., (8) ein, zu deren Bestimmung die Messungen genügendes Material bieten. Indem ich auf diese Methode eingehe und mich ganz an die Gruppeneintheilung von Prof. Schur halte, will ich im Folgenden durch eigene Berechnung meine obige Behauptung begründen, und dazu zunächst über meine Abweichungen von der Schur'schen Rechnung einige einfachen Bemerkungen vorausschicken.

Es ist von vornherein klar, dass sich die Messungsfehler ihren wirklichen Werthen nach überhaupt nicht bestimmen lassen. Vielmehr bleibt ein der gemessenen Distanz pro. portionaler Fehler auf jeden Fall unbestimmbar, da er nur die Maasseinheit, den Scalenwerth, verändert, sich also völlig mit der Bestimmung desselben verbindet und gar nicht davon trennen lässt. Er hat infolgedessen auch absolut keinen Einfluss auf das Resultat der Messungen, und die Bestimmung der systematischen Fehler von Distanzmessungen hat daher nur ihre Abweichungen von einem der gemessenen Distanz proportionalen Verlauf zu liefern. ${ }^{*}$. Man kann diesen systematischen Fehlern, indem man ihnen eine unbestimmte, der Distanz proportionale Grösse hinzufügt, rein äusserlich verschiedene Formen geben, man kann z. B. den Fehler für eine beliebige Distanz (bei Prof. Schur 7200") nur $s=0^{\prime \prime}$ ist ausgenommen - zum Verschwinden bringen; Weise dar. In welcher Weise sich dieses willkürliche Element in der Rechnung nach der Schur'schen Methode Geltung verschafft, übersieht man leicht. Es lassen sich aus den Bedingungsgleichungen nicht alle 8 Fehler bestimmen, sondern nur 7 von ihnen als Functionen des achten unbestimmt bleibenden, und die Abhängigkeit von diesem achten ist dem Verhältniss der beiden zugehörigen Distanzen proportional, Für verschiedene Werthe dieses achten Fehlers alle diese Fehlercurven stellen die Messungen in gleicher

ergeben sich aequivalente Fehlercurven. Z. B. folgt, wenn diese Fehlercurve geradlinig verläuft, daraus von selbst die Existenz eines constanten Fehlers, und wenn ein solcher existiren soll, muss die Fehlercurve eine gerade Linie sein.

Wir wollen nun unsere Rechnung so führen, dass wir die 7 ersten Feblerwerthe (1), (2), ., (7) als Functionen des letzten, unbestimmt gelassenen, darstellen.

Die zweite Abweichung von der Schur'schen Rechnung ist die, dass wir für jede der gemessenen 28 Distanzen $s_{i, k}$ die entsprechende Bedingungsgleichung ansetzen und so 28 Gleichungen mit 15 Unbekannten (7 Elementar-Distanzen und 8 Fehlerwerthe), also $x_{3}$ überschüssige erhalten; Prof. Schur hingegen setzt die gemessenen Distanzen auf alle mögliche Weise zur Enddistanz $s_{\mathrm{I}, 8}$ zusammen und erbält so 55 Gleichungen für die 8 Fehlerwerthe, also 47 überschüssige. Natürlich ist das nur scheinbar, da diese Gleichungen in vielfacher Abhängigkeit von einander stehen. Den Regeln der Ausgleichungsrechnung entspricht nur das erste Gleichungssystem.

Als Resultat meiner Rechnung, auf dessen Anführung ich mich beschränken will, folgt:

$$
\begin{aligned}
& (\mathrm{r})=+0.268+0.054(8) \\
& (2)=+0.229+0.118(8) \\
& (3)=+0.166+0.175(8) \\
& (4)=+0.143+0.293(8) \\
& (5)=+0.025+0.428(8) \\
& (6)=+0.100+0.555(8) \\
& (7)=-0.023+0.827(8)
\end{aligned}
$$

woraus sich für die 3 Werthe: $-0 . " 25$, o."0o und +0.25 von (8) die folgenden Fehlertabellen ergeben.

$\begin{array}{ccccc}\text { Distanz } & \text { Fehler } & \text { I } & \text { II } & \text { III } \\ 358^{\prime \prime} & (1) & +0.25 & +0.27 & +0.28 \\ 715 & (2) & +0.20 & +0.23 & +0.26 \\ \text { I176 } & (3) & +0.12 & +0.17 & +0.21 \\ 1982 & (4) & +0.07 & +0.14 & +0.22 \\ 2894 & (5) & -0.08 & +0.03 & +0.13 \\ 3710 & (6) & -0.04 & +0.10 & +0.24 \\ 5750 & (7) & -0.23 & -0.02 & +0.18 \\ 6728 . & (8) & -0.25 & 0.00 & +0.25\end{array}$

*) Darum wäre es eigentlich wünschenswerth, die gesammte Untersuchung der systematischen Fehler an die directe Messung in Scalentheilen vor ihrer Verwandlung in Winkelmaass anzukniupfen und zu untersuchen, ob die in Scalentheilen ausgedruickten Messungswerthe den gemessenen Distanzen wirklich proportional sind oder ob Abweichungen - d. h systematische Messungsfehler - auftreten. Man würde so die systematischen Fehler wie naturgemäss in Scalentheilen ausgedriickt erhalten, wobei es für die innere Uebereinstimmung gleichgültig ist, was als Scalenwerth zu Grunde gelegt wird (ob I" oder $\mathrm{I}^{\prime}$, der mittlere Fehler in Scalentheilen ausgedrückt bleibt derselbe). Dann erst, wenn die Distanzmessungen von ihren systematischen Fehlern befreit in einem wirklichen, einheitlichen Maass dargestellt sind, wïrde durch ihren Vergleich mit Meridianbeobachtungen der Winkelwerth dieser Maasseinheit, der Scalenwerth, zu ermitteln sein. 
Die letzte Columne zeigt mit einer von mir kaum erwarteten Deutlichkeit, insbesondere wenn man die Unsicherheit der Bestimmung berücksichtigt, die Existenz eines constanten Messungsfehlers.

Mit der unter I gegebenen stimmt nun die Schur'sche Fehlertabelle, wie es bei richtiger Rechnung ja auch sein muss, im grossen und ganzen überein, und Prof. Schur hat selbst gezeigt, dass seine Tabelle im Wesentlichen auf einen constanten Messungsfehler führt, indem er sie in die Form $p+q x+r x^{2}$ brachte. Hierin ist $p$ nichts anderes als der von mir $c$ genannte constante Messungsfehler, $q$ die gleichgültige Verbesserung des Scalenwerthes, $r$ aber hat wenig reelle Bedeutung, wie ich in meiner früheren Arbeit, mit deren $d$ es identisch ist, gezeigt habe. Nur die beiden Nullwerthe für $s=0^{\prime \prime}$ und $s=7200^{\prime \prime}$ fehlen in meiner Tabelle; aber diese Distanzen reichen ja über die Grenzen der vorliegenden Messungen hinaus, über sie können die Messungen also auch nichts aussagen. $\mathrm{Ob}$ die beiden, nach. träglich hinzugefügten Nullwerthe der Schur'schen Fehlertabelle zum Vortheil gereichen, mag dahingestellt bleiben. In den Grenzen der vorliegenden Messungen - und darum kann es sich doch nur handeln, - stimmen daher beide Fehlertabellen überein und innerhalb dieser Grenzen ist daher ein constanter Fehler wahrscheinlich gemacht.

Wir vergleichen noch die nach der Schur'schen Methode, d.h. ohne Annahme über die Form des Fehlers, abgeleitete Fehlertabelle mit den auf Grund der Formen $c$, $d\left(\frac{s}{1000^{\prime \prime}}\right)^{2}, e\left(\frac{1000^{\prime \prime}}{s}\right)$ sich ergebenden, wobei wir das verfügbare lineare Glied so wählen, dass Fehler (8) verschwindet. In der folgenden Tabelle sind unter. Sch. die nach Schur's Methode oben abgeleiteten, unter $\bar{A}, \mathrm{~B}, \mathrm{C}$ die folgenden Werthe gegeben:

$$
\begin{aligned}
& \text { A: }+0.232-0.0345 \frac{s}{1000 "} \\
& \text { B: }-0.0113\left(\frac{s}{1000 "}\right)^{2}+0.0760 \frac{s}{1000 "} \\
& \text { C: +0." I } 6\left(\frac{1000 "}{s}\right)-0.0026 \frac{s}{1000 "}
\end{aligned}
$$

$\begin{array}{rrrrr}\text { Distanz } & \text { Sch. } & \text { A } & \text { B } & \text { C } \\ 35^{\prime \prime} & +0.27 & +0.22 & +0.03 & +0.32 \\ 715 & +0.23 & +0.21 & +0.05 & +0.16 \\ 1176 & +0.17 & +0.19 & +0.07 & +0.10 \\ 1982 & +0.14 & +0.16 & +0.11 & +0.05 \\ 2894 & +0.03 & +0.13 & +0.13 & +0.03 \\ 3710 & +0.10 & +0.10 & +0.13 & +0.02 \\ 5750 & -0.02 & +0.03 & +0.06 & +0.01 \\ 6728 & 0.00 & 0.00 & 0.00 & 0.00\end{array}$

Der constante Fehler schliesst sich also den Werthen Sch. am besten an, der quadratische ist gänzlich unbrauchbar; besser stimmt der der Distanz umgekehrt proportionale, indessen ist der starke Anstieg bei den kleinen Distanzen, der für diese Form entscheidend ist, durch die Beobachtungen nicht genügend gerechtfertigt.

Endlich wollen wir die Werthe für die ElementarDistanzen vergleichen, wie sie sich bei Anwendung der 4 Fehlertabellen ergeben, da doch die Bestimmung dieser Werthe der Zweck astronomischer Messungen ist. Die folgende Tabelle giebt die Abweichungen von den nach Schur's Methode sich ergebenden Werthen, wobei unter $D$ die ohne Annahme systematischer Fehler folgenden Werthe hinzugefügt sind.

$\begin{array}{cccccc}\text { Distanz } & \text { Sch. } & \text { A } & \text { B } & \text { C } & \text { D } \\ s_{1,2} & 3141.909 & -0.004 & +0.057 & +0.052 & +0.1122 \\ s_{2,3} & 813.370 & -0.027 & -0.029 & -0.027 & -0.038 \\ s_{3,4} & \text { 1228.407 } & +0.039 & +0.076 & +0.014 & +0.05^{2} \\ s_{4,5} & 656.435 & +0.015 & +0.010 & -0.016 & -0.005 \\ s_{5,6} & 386.274 & -0.004 & -0.031 & -0.014 & -0.039 \\ s_{6,7} & 316.758 & -0.009 & -0.043 & -0.002 & -0.049 \\ s_{7,8} & 371.190 & -0.008 & -0.039 & -0.008 & -0.045\end{array}$

Sonach folgt auch nach der Schur'schen Methode mit erheblicher Sicherheit, dass bei seinen Controllmessungen im Wesentlichen ein constanter Messungsfehler vorliegt. Allerdings soll keineswegs behauptet werden, dass nunmehr seine reelle Existenz absolut sicher gestellt und er die einzige Ursache der zu Tage getretenen Schwierigkeiten sei; vielmehr erscheint nach den obigen Tabellen z. B. ein theilweises Mitwirken eines Fehlers von der Form $e s^{-1}$ nicht als völlig ausgeschlossen. Der Zweck meiner Untersuchungen war nur, zu zeigen, dass die Annahme eines constanten Messungsfehlers geeignet ist, die systematischen Fehler der Schur'schen Controllmessungen in einfachster und befriedigendster Weise darzustellen.

Bezüglich der kürzlich (A. N. Nr. 3406) erschienenen, meine Arbeit betreffenden Bemerkung von Herrn Dr. Battermann erwähne ich, dass sich dieselbe offenbar nur auf die Bestimmung des Scalenwerths bezieht, die mir in meiner Arbeit ganz fern lag. Sein Vorschlag bezüglich der Vermeidung der aus der Ungenauigkeit der Meridianbeobachtungen herrührenden Unsicherheit der Projectionsfactoren betrifft wohl die Ableitung des Scalenwerths, kann aber bei dem Thema meiner Arbeit, der Untersuchung der systematischen Fehler heliometrischer Distanzmessungen, gar keine Verwendung finden. Bei dieser muss die Unsicherheit der Projectionsfactoren als die zufälligen Messungsfehler vergrössernd angesehen werden und lässt sich jedenfalls in keiner Weise úmgehen. Sonach lag für mich kein Grund vor, auf diesen Punkt hinzuweisen, da meine Resultate dadurch nicht im Mindesten beeinflusst werden konnten.

Fritz Cohn.

Königsberg, 1897 Februar.

Inhalt:

Zu Nr. 34I I. G. Ciscato. Osservazioni di pianeti. 33. - Leo Brenner. Veränderungen auf dem Mars I896. 4I. - V. Cerulli. Note su Marte. Dall' Ottobre 1896 al Gennaio 1897. 43. - Fritz Cohn. Bemerkungen zu Herrn Prof. Schur's Abhandlung 》Neue Untersuchungen über den Verlauf der systematischen Fehler bei Distanzmessungen in A. N. 3399. 45

Geschlossen 1897 März sg. Herausgeber, in Vertretung: H. Krentz, Druck von C. Schaidt. Expedition: Kiel, Wrangelstrasse 6. 